\title{
ATOM- A Low-Cost Mobile Manipulator for Research and Testing
}

\author{
Abhijit Makhal \\ Indian Institute of Information \\ Technology \\ Deoghat, Jhalwa
}

\author{
Manish Raj, Karan Singh, \\ Rajan Singh \\ Indian Institute of Information \\ Technology \\ Deoghat, Jhalwa
}

\author{
Pavan Chakraborty, PhD., \\ G.C.Nandi \\ Indian Institute of Information \\ Technology \\ Deoghat, Jhalwa
}

\begin{abstract}
This paper represents the technique of developing a ROS (Robot Operating System) based low cost mobile manipulator. Additionally, the contemporary commercial and custom ROSbased mobile manipulators have been discussed. In this paper, we have discussed how the performance of the high-end mobile manipulation platforms can be partially achieved by a low-cost custom made module implementing the basic packages of ROS. The architecture and till to date development on the platform-ATOM (Autonomous Testbed of Mobile Manipulation) has been also shown.
\end{abstract}

\section{General Terms}

Arm manipulation, Low-cost mobile manipulator

\section{Keywords}

ROS, Mobile Manipulators, Mapping, Arm manipulation

\section{INTRODUCTION}

It has always been a Roboticist's dream to create a robot that can socially interact with the human being, manipulate or fetch objects, roam around daily-life cluttered environments like in home, office even that in kitchen scenes autonomously. In more general terms we always wanted a robot beside us which help our work as an assistant by bringing our daily-life objects with only just a single voice command e.g. - "bring me the pen" without any lower level programming intervention. As this is an easily understandable and performable command for a little brother or a friend, it is not that easy for a robot. The robot should have the touch of various intelligent techniques namely natural language processing, image processing, localization and mapping techniques, arm manipulation techniques and many more which took us several years to gain knowledge about them and to implement them in the modules.

But only the qualities of manipulating objects or generating maps autonomously are not sufficient for a robot to become socially acceptable. In 1970 Masahiro Mori introduced the concept of "Uncanny Valley" [1] which states that "a person's response to a humanlike robot would abruptly shift from empathy to revulsion as it approached, but failed to attain, a lifelike appearance". He hypothesized that as robots become more humanlike, they appear more familiar until a point is reached at which subtle imperfections of appearance make them look zombie.

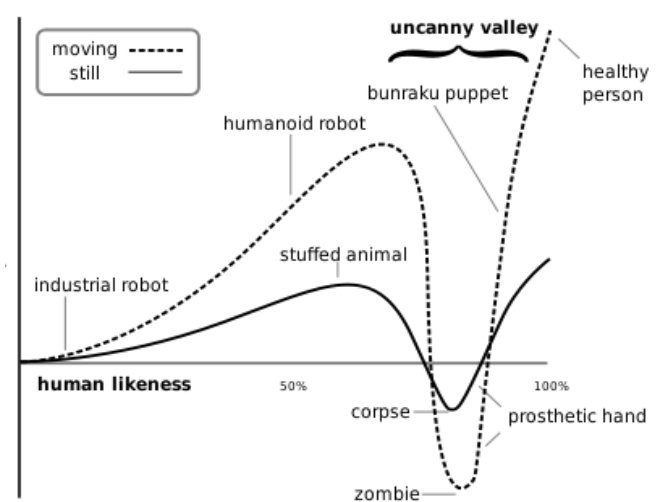

Fig 1: The Conceptual illustration of Mori's Uncanny valley [1]

For the past few decades, robotics engineers around the world have indulged themselves in fulfilling the dream of providing those previously mentioned capabilities in their robots keeping those constraints in mind. At the end of the last century the Japanese scientists amazed us with the creation of those gorgeous robots namely the ASIMO series. Biped locomotion is one of the most researchable topics in the field of robotics and which makes the humanoids different from the other robots. As the desired robot to be working in the office or room environment, we can think over the robots which have a wheeled mobile base with navigation capabilities, arms with manipulation capabilities, and a sensor-architecture with interaction capabilities- a mobile manipulator. As the scale and scope of the hardware varies in different organizations, the process of creating codes for those modules becomes much daunting with the context of truncation in the reusability. Since the required breadth of expertise is well beyond the capabilities of any single researcher, robotics software architectures must also support large-scale software integration efforts-ROS [2]. The advantageous functionality of ROS is the reusability, easy implementation, world-class technology and network-based architecture. Though the capability of easy-implementation -You would not understand ROS properly until you see your robot moving in-front of you using ROS. 
The paper is structured as follows. In Section II we have discussed the previous work related to commercial modules using ROS, after that we have tried to summarize the custom modules which are currently using ROS. Then the low cost custom modules have been discussed. In section III we have discussed the hardware architecture of the low cost platform ATOM. Then in Section IV, the still up-to-date development and results in the module using ROS have been shown. It is been concluded with the future plan and program of development of the team on ATOM. such features in a single platform. The PR2 contains an omnidirectional base, telescopic spine, modular head with panning and tilting capability and two 7-dof arms. The perception system contains one 5-Megapixel Global Shutter Color Gigabit Ethernet Camera (1-Megapixel), Wide-Angle Global Shutter Color Stereo Ethernet Camera, Narrow-Angle Global Shutter Monochrome Stereo Ethernet Camera, LED Texture Projector Triggered with Narrow-Angle Stereo Camera, Tilting Hokuyo UTM-30LX Laser Scanner, Hokuyo UTM-30LX Laser Scanner for the base, Microstrain 3DM-
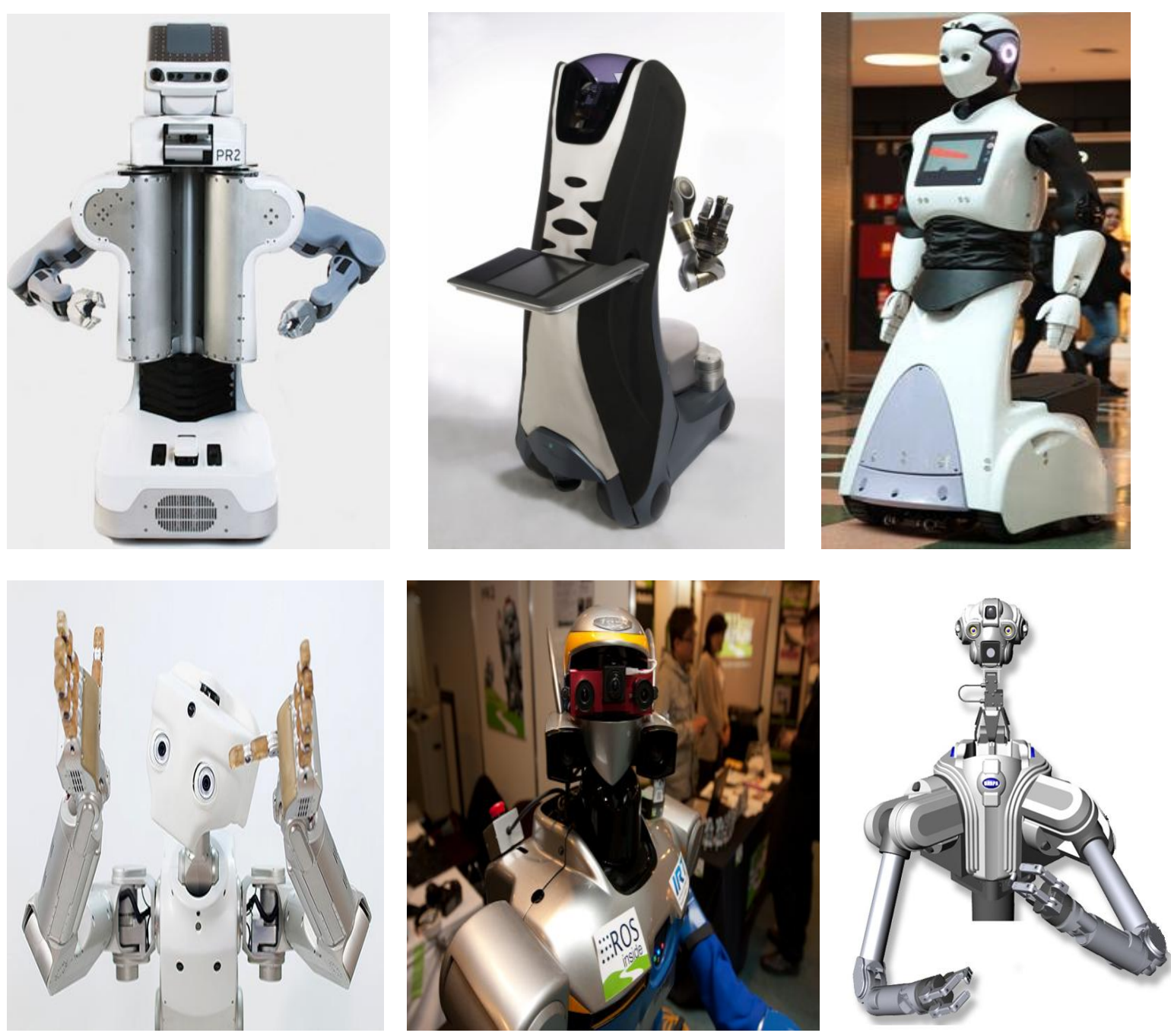

Fig 2: Contemporary Robots using ROS.(From left to right, top to bottom) a) PR2 from Willow Garage [3] , b) Fraunhofer IPA Care-O-Bot [4], c) REEM from PAL Robotics, d) Meka M1, e) Khwada HRP-2, f) ARM project from DARPA [6]

\section{Previous Work}

From the past few decades robot persons are involved in creating such a mobile manipulation platform which can combine tasks of interacting naturally, manipulating objects independently, localizing and wandering autonomously and can be a personal assistant in our home, kitchen even that in office environments.

The dream came true when the Willow Garage Personal Robot (PR2) [3] released in the market combining
GX2 IMU, Three-Axis Accelerometer, Fingertip Pressure Sensor Arrays, Calibration LEDs. As the recent research findings of Willow Garage suggests, the platform is capable of many complex tasks in cluttered environment autonomously such as folding clothes, fetching beer, running 3 days with self charging and lots more. But as the cost is so high, it is still not available for maximum robotics engineers who are not associated with those institutes or organizations who can afford those platforms. Though PR2 provides the most suitable example for implementation of ROS, people 
who wish to work on the platform can still perform their programs or research findings through the simulator provided by ROS-Gazebo. The PR2 platform is been shown in Fig.2.a.

Another popular ROS based robot platform which is commercially available is the Fraunhofer IPA Care-O-Bot [4] [5]. The system is generally built for assisting elderly or disabled persons in home environments. The platform mainly consists of an omnidirectional base, Schunk LWA3 arm and gripper with tactile sensors, pan-tilt torso and one tray for carrying objects. The perception system consists of two SICK S300 laser scanners, one Hokuyu URG-04LX laser scanner, two AVT Pike $145 \mathrm{C}, 1394 \mathrm{~b}, 1330 \times 1038$ (stereo circuit), MESA Swissranger 4000 or Microsoft Kinect. The Care-OBot platform has also shown some soothing results and been proven as one of the pioneer platform in the advancement of mobile manipulation platform. The Care-O-Bot is shown in Fig 2.b. DARPA ARM (Autonomous Robotic Manipulation) [6] is another popular platform using ROS. This platform is been created by Defense Advanced Research Projects Agency. valued components. Despite of the high valued commercial robots, the institutes around the world have also started to create their own modules where they can execute their research outcomes. Some of the most gorgeous custom mobile manipulators also came from the world-renowned institutions like Stanford University, TUM Munich, Georgia Tech, Cornell University and many more. The examples of this kind of mobile manipulators are STAIR1 robot from Stanford University, TUM-Rosie from TUM-Munich, ELE (Fig 4.a) and Cody from Georgia Tech, Blue, Panda and Polar from Cornell University. Fig [3] shows few of them. Currently the TUM-Rosie which is one of the pioneer creations in the project ROBOHOW.COG [7] amazed the world by performing complex everyday tasks by knowledge-enabled and plan-based approach using web-enabled and experiencebased learning techniques. The long term goal of the STAIR [8] project is to create a robot that can navigate in home and office environments, pick up and interact with objects and tools (including carrying out more complex tasks such as unloading a dishwasher), and intelligently converse with and
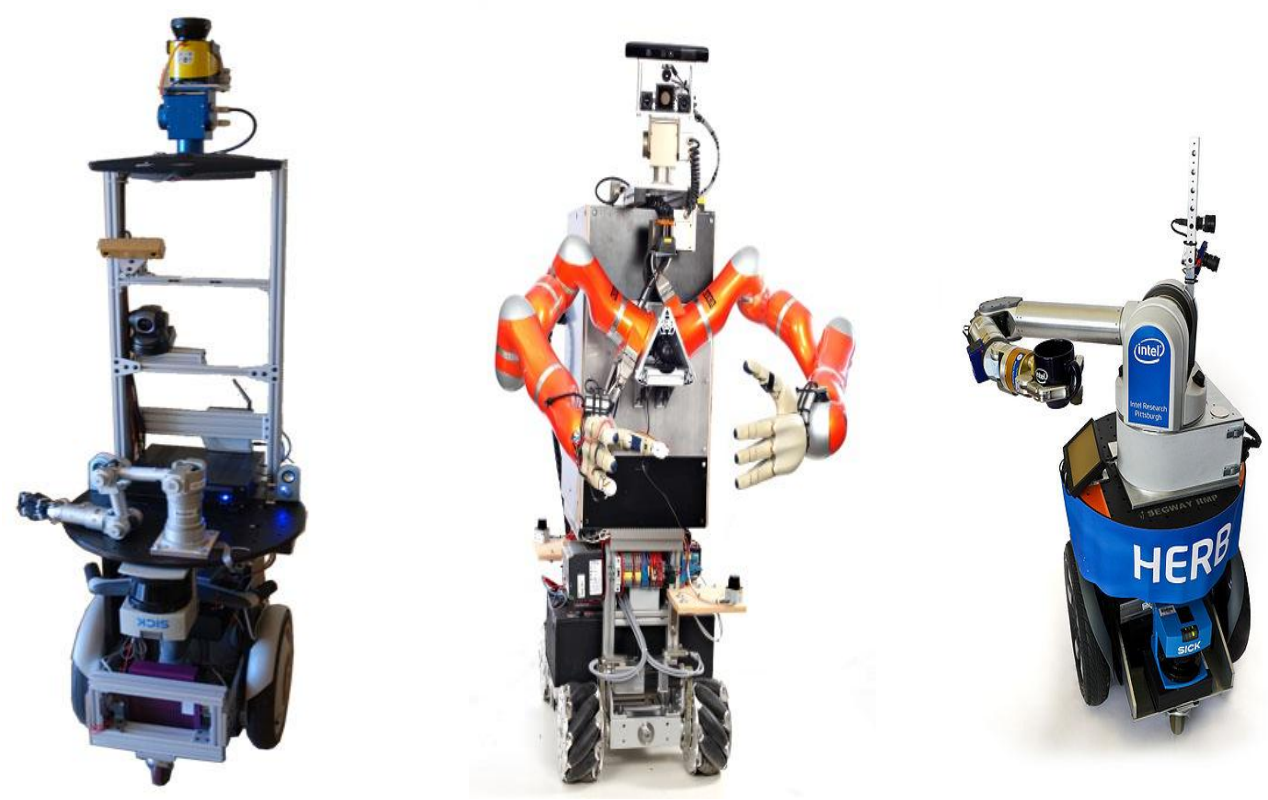

Fig 3: Custom-built mobile Manipulators using ROS (From left to right) a) STAIR [7] project from Stanford, b)TUMRosie from TUM-Munich, c)HERB from Intel Research [9]

The robot had two Barrett WAM arms with seven degrees of freedom, two three-fingered Barrett hands with pressure sensors and torque sensors, a BumbleBee2 stereo camera, a Prosilica high resolution camera, an SR4000 Swiss Ranger infrared camera, and a four degree of freedom neck. This robot is been presented in the Fig.2.f.

Other Mobile manipulation platforms that are been developed with joint collaboration with industrial background are REEM service robot from PAL Robotics (Fig 2.c) and Meka M1(Fig 2.d) mobile manipulator. Recently Khwada HRP-2(Fig 2.e) of JSK lab, Japan also integrated into ROS.

Most of the system mentioned previously is mainly created in joint collaboration with the industry or the organizations with sufficient funding for affording those high help people in this environment. Intel Research Pittsburg, with collaboration with Robotics University at Carnegie Mellon created the mobile manipulator platform HERB (Home Exploring Robotic Butler) which has been demonstrated in a variety of real-world kitchen tasks, such as opening refrigerator and cabinet doors, finding and collecting coffee mugs, and throwing away trash [9][10].

MAXWELL [11][12] has first shown how a reliable mobile manipulator platform can be created using a Kinect, ArbotiX RoboController, Dynamixel servos, and a custom differential-drive base. Maxwell sports a large 16x16" version Armadillo base with motors that support a $201 \mathrm{~b}$ payload at speeds up to $0.5 \mathrm{~m} / \mathrm{s}$. A Hokuyo URG-04LX-UG01 has been mounted on the base for range measurement. The head has two AX-12 servos for pan and tilt motion. 

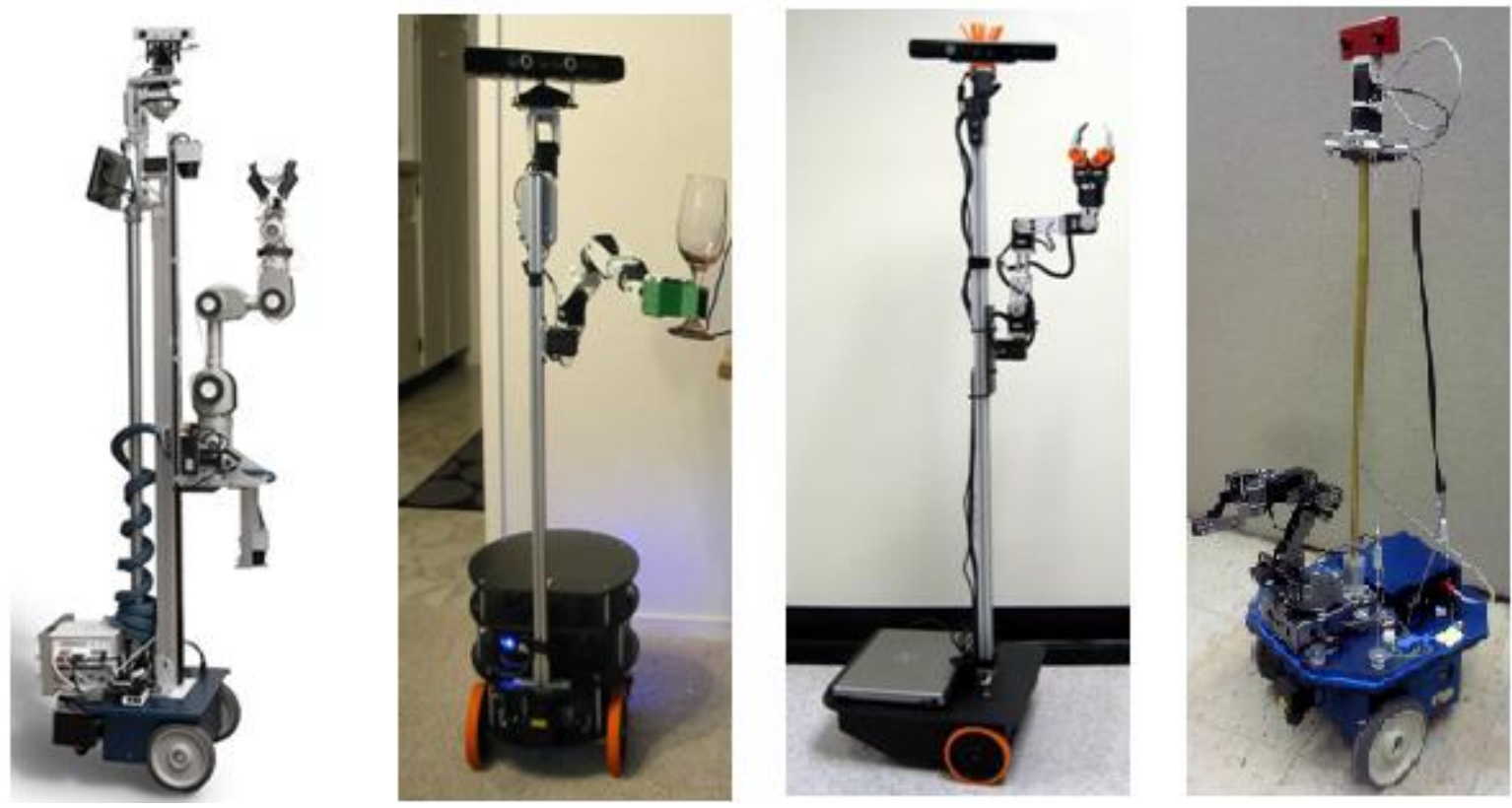

Fig 4: Low cost custom mobile manipulators that showed promising results. a)EL-E from George Tech Healthcare Robotics Lab, b) Pi-robot [12], c) Maxwel [11], d) Wubble

The arm is constructed by 2 EX-106 (shoulder lift and elbow flex), 2 RX-64 (shoulder pan and wrist flex), and 3 $\mathrm{AX}-12 \mathrm{~s}$ (one for wrist roll, and two to form a gripper). A piece of T-slot extruded aluminum is been used as the vertical track and a 20" linear motor to move the arm up and down. As the Microsoft Kinect requires an external power supply, Maxwell is been equipped with an Asus Xtion Pro Live 3D camera which takes power from the processing system. Maxwell won 1st place in the 2011 AAAI Small Scale Manipulation Challenge. Maxwell is shown in Fig 4.b.

Other pioneer in low cost custom mobile manipulator are the Pi-robot[13] and the Wubble [16]. Pi-robot is highly inspired from the MAXWELL and the Turtlebot [14]. It has got a Hokuyo URG-04LX-UG01 scanning laser rangefinder and a Microsoft Kinect for range and depth processing.

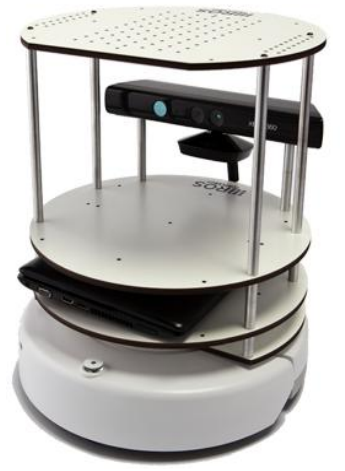

Fig 5: Turtlebot Robot

The wheels are 5" BaneBots wheels connected with Pololu 100:1 220 oz-in 100 RPM drive motors. The DC motors is been controlled by Polulu 30A motor driver.
Serializer microcontroller from Robotics Connection is been used to monitor overall battery voltage and current from the Phidgets sensors .The arm of the Pi-robot is a standard turtlebot arm created AX-12 motors. Total weight of the robot is $17.4 \mathrm{lbs}(7.9 \mathrm{~kg})$. Wubble robot is been shown in Fig 4.d.

\section{System Description}

In this section the very aspect of ATOM has been described. As the system is currently under development, it can be considered that in near future it would be proved to be highly efficient system for advancement of mobile manipulation platform in social environment and will be a guide for the future researchers who wish to create their own low-cost module.

There are 5 main modules of the platform: i) Base and Torso, ii)Head, iii)Arms and Grippers, iv)Perception System, v)Processing System

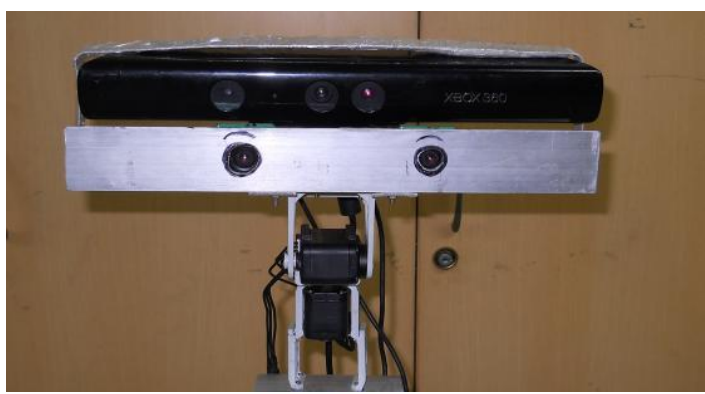

Fig 6: Perception System of ATOM

The robot is been provided with a non-holonomic base with a differential drive wheel system. At the front and the rear there are 2 castor wheels. The driving wheels are of 4 " radius. The 
drive system consist two 30:1 85RPM 37DL Gear Motors with Shaft Encoder. Currently many mobile robots namely Clearpath Robotics Husky, Neobotix mp-500, Videre Erratic, I-robot roomba are using ROS for navigation. The mostly available and suitable low cost platform is Turtlebot [Fig 5]. It is a module consists of a cylindrical base attached on a Irobot Roomba. Technical development has been also implemented by attaching an arm to the module. The concept of the cylindrical base created by 3 circular plates attached by 11.8 " and 2.3" standoffs has been inherited from Turtlebot. The processing system is situated in the second plate of the robot. Third plate is attached with the torso of the robot. The torso is created by custom-modified aluminium plates. It is been connected with the arm system and the head.

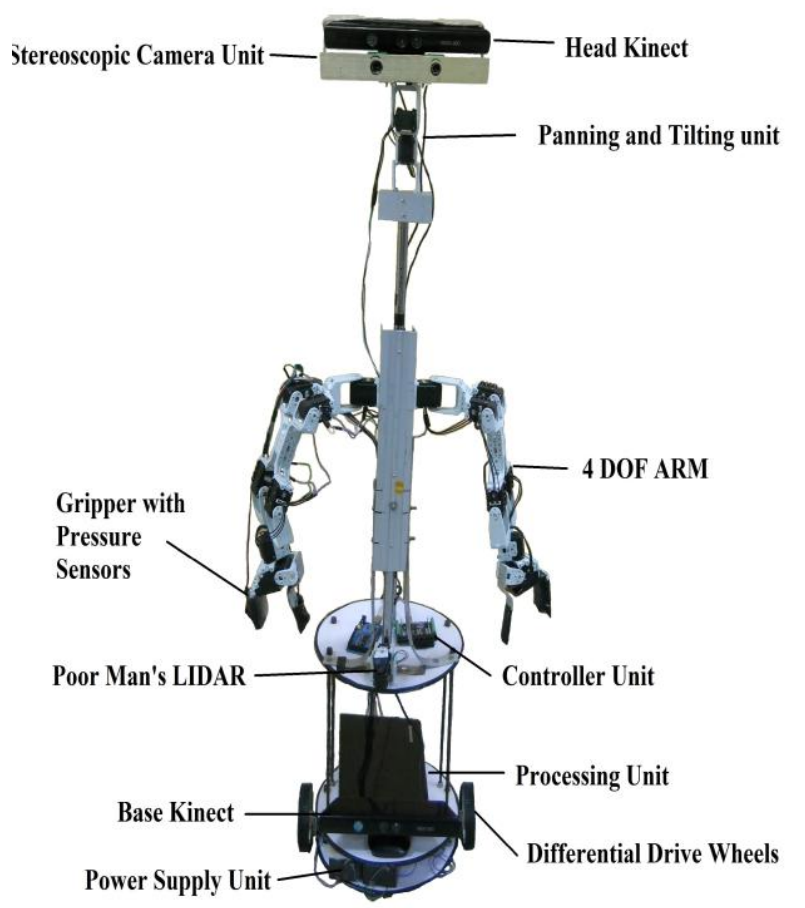

Fig 7: Different systems of our the low-cost custom Robotic platform-ATOM

The base of the system is been shown in Fig 8.a. ATOM is equipped with a panning and tilting head which is capable of $48^{\circ}$ vertical and $180^{\circ}$ horizontal movement. Two AX-12 motors are responsible for this panning tilting capability.

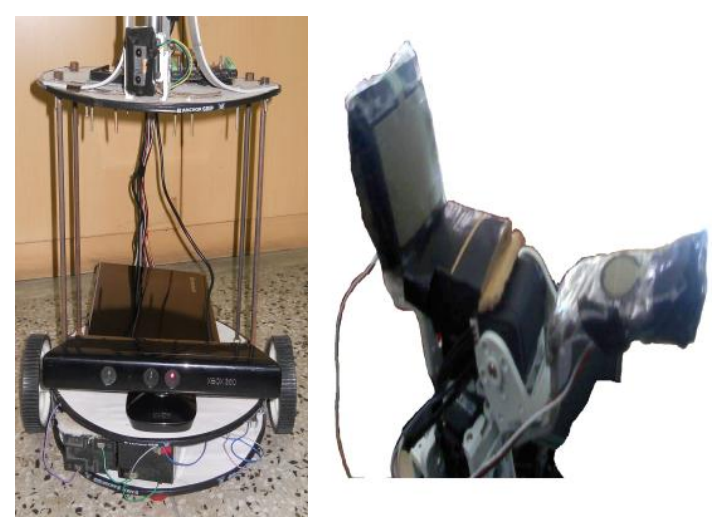

Fig 8: Different modules of ATOM (From left to right a) The Base, b) The gripper with pressure sensors
The workspace of the arms of ATOM is 19". The arms are been created by AX-12 motors. Our finding through iteration process suggests that single AX-12 motor in shoulder_lift and wrist_flex joint is not sufficient for manipulating objects weighted more than 100 grms $(.22 \mathrm{lbs})$. So the robot is been provided with two AX-12 motors in the shoulder-lift and wrist_flex joint for both the arms. The arms are been shown in Fig 9. A single-sided moving gripper with an AX-12 motor which has $10 \mathrm{~cm}$ (3.93") aluminium sheet for thumb and 2.7" * 2.7" plate for palm has been created. The gripper also contains pressure sensors which can measure the force enforced by the grippers. The gripper has been shown in the Fig 8.b.

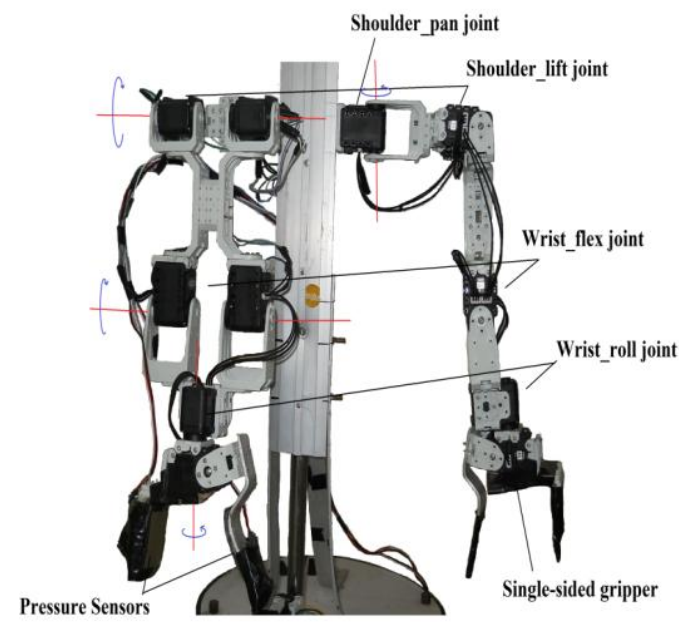

Fig 9: The arm system of ATOM. Joints of the two DOF arms also have been shown.

The current structure of the robot contains a Microsoft Kinect (base_kinect) in the base for range measurements. The panning tilting head is provided with two Logitech C600 720p HD cameras for the stereo system. The head is also mounted with a Microsoft Kinect (head_kinect) which also provides depth images using the IR system. ATOM has also got a stereo microphone for capturing audio information and interacting naturally with the user. The gripper of the robot contains phidget sensors in the palm and the thumb for measuring pressure enforced by the gripper on the objects to be manipulated. We have adopted a SONY VAIO VPCCB15FG laptop as the main processing unit containing Intel second generation $2.30 \mathrm{Ghz}$ Intel Core i5-2410M (Sandy Bridge) processor, 4GB DDR3 RAM, on-board Intel HD graphics and AMD Radeon 6630M graphics with $1 \mathrm{~GB}$ of RAM. We have created a custom board for interfacing the USB ports. The power system of ATOM contains AMARON lithium battery of $12 \mathrm{v}$ and $7.5 \mathrm{Amp}$. The power system drives the two differential drive wheels in base, the total $14 \mathrm{AX}-12$ motors in the arms, 2 AX-12 motors in the neck for panningtilting, the Kinect in the base and the kinect in the head. A custom circuit has been created for powering the two kinect with $12 \mathrm{~V}$ power source.

\section{Result and Discussion}

For navigating the robot in indoor environment, the navigation package of ROS has been implemented using a Microsoft Kinect instead of using a LIDAR system. 

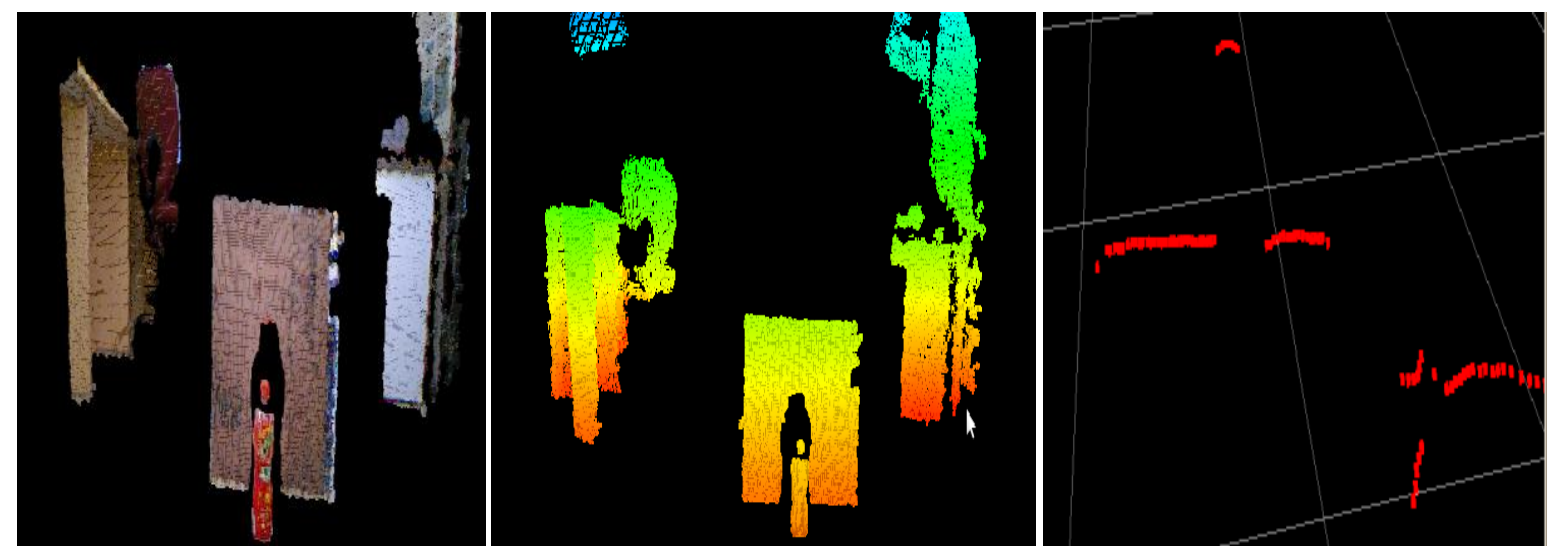

Fig 10: (From left to right) The point cloud image taken by the base kinect, b) The depth image, c) The Laser scan image converted from the point cloud image

Previously a PML (Poor Man's Lidar) [15] has been created using a Sharp GP2Y0A02YK0F IR sensor mounted vertically on a $\mathrm{AX}-12$ motor. The conventional laser range finder system gives around 650 points in single 240 degree sweep, where the PML system can only provide around 24 points in a 180 degree sweep. In the current system of ATOM, the Kinect in the base takes the depth information and converts it as laser scan using Pointcloud_to_laserscan package. This package creates fake laserscan from point cloud by horizontally slicing the image and considering the closest distance in each column. Fig.10 shows the point cloud image, depth image and laserscan images taken from the base_kinect. In Dead Reckoning phase the robot creates a $2 \mathrm{~d}$ map of the environment by the laser scans acquired by the base_kinect. The first map created by ATOM in our corridor environment has been shown in Fig 11.a. The differential drive wheels been interfaced by an Arduino Mega 2560, which takes cmd_vel msg from the higher level interface and sends back odom using the rosserial library which is the interface library of Arduino using ROS. Combining them with the single axis gyro measurement, odom combined has been created, which became highly helpful in localizing ATOM in its map. The two Logitech C600 720p HD cameras of the stereo system has been calibrated using the stereocameraV412 package of ROS and depth images from the two cameras has been extracted by stereo_image_proc package which takes the two images from the left and right cameras and performs rectification and demosaicing of raw stereo camera image pairs.

The two 4 DOF arms of ATOM are been manipulated using arm_navigation package of ROS. The AX-12 motors have been interfaced by usb2dynamixel device which publishes the motor_states and subscribes to the joint_trajectory_msgs using the dynamixel_motor package. With the introduction of Planning Description Configuration Wizard, ROS has simplified the process of navigating the arm of the robot. The wizard takes only the URDF (Unified Robot Description Format) of the robot and outputs various packages including move_arm, ompl_planning, planning_environment, planning_components_visualizer, trajectory_filter_server, constraint_aware_kinematics,planning_scene_warehouse_vie wer which are highly helpful in navigation the arms. A follow_controller node has been created which is an action interface to the controller in the form of a ROS action, that takes in a trajectory command expressed as a series of joint angles and sends the appropriate low-level commands to the low-level controller. The URDF of ATOM has been shown in Fig 11.b. The state of the motors has been published by the robot_state_publisher . The transformations between different co-ordinates frames of the robot is been maintained by a transformation tree of the $t f$ package. Phidget sensors are been interfaced with the robot using the phidget_ros package of Arizona State University.
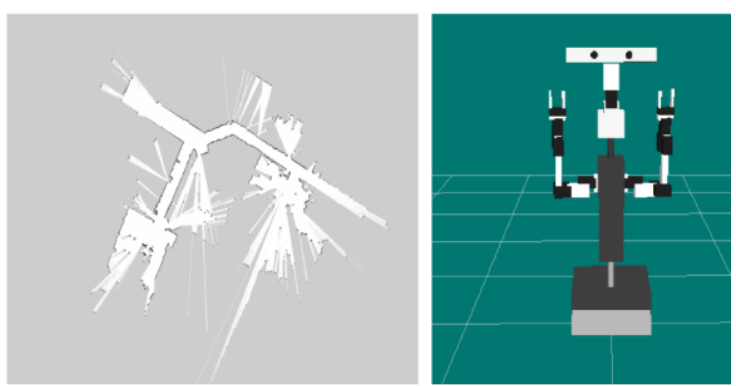

Fig 11: A) The first map created by ATOM in corridor environment, b) The visualization of the URDF of ATOM in RVIZ

As the range of the Microsoft Kinect is around $6 \mathrm{~m}$, the laser scan created by the system cannot find objects beyond the range. Traditional LIDAR system takes the range of $30 \mathrm{~m}$. We need to modify our system using a Laser Range Finder. For the arm navigation purpose, ROS creates a collision map by the tiltable laser range finders for safe arm trajectory. As we are lacking of LIDAR system, the point cloud created by the Kinect does not provides suitable results in creating collision maps. As the whole powering system relies on a single battery, the system is only capable of operating for just 20 minutes. Currently ATOM weights around 17.85 1bs (8.1 $\mathrm{Kg}$ ). The robot will be increased in weight with addition of extra powering system- which will affect the differential driving system. The arm system is only created by AX-12 motors, which is not sufficient not pick and place tasks. As the robot's workspace is not suitable for table-top manipulation, we are trying to improve the DOF and dexterity of the arm by providing more joints and high torque servo motors. As the grippers only contain the pressure sensors, we need some sensor array which can also detect slip detection which will be highly beneficial in grasping task. 
Table 1. Comparison between different systems of different Low-cost Mobile Manipulators

\begin{tabular}{|c|c|c|c|c|}
\hline $\begin{array}{c}\text { Low-cost } \\
\text { mobile } \\
\text { manipul }\end{array}$ & Head & Arm & $\begin{array}{l}\text { Base- } \\
\text { sensor }\end{array}$ & Base \\
\hline Maxwell & $\begin{array}{l}\text { Microsoft } \\
\text { Kinect with } \\
2 \mathrm{AX}-12 \\
\text { for panning } \\
\text { and Tilting }\end{array}$ & $\begin{array}{c}5 \text { Degree } \\
\text { Of } \\
\text { Freedom- } \\
2 \text { EX-106 } \\
, 2 \text { RX-64, } \\
\text { and } 1 \mathrm{AX}- \\
12\end{array}$ & $\begin{array}{c}\text { Hokuyo } \\
\text { URG- } \\
\text { 04LX- } \\
\text { UG01 }\end{array}$ & $\begin{array}{c}\text { Armadillo } \\
\text { base with } \\
\text { motors } \\
\text { that } \\
\text { support a } \\
\text { 20lb } \\
\text { payload }\end{array}$ \\
\hline Pi-robot & $\begin{array}{l}\text { Microsoft } \\
\text { Kinect with } \\
2 \mathrm{AX}-12 \\
\text { for panning } \\
\text { and Tilting }\end{array}$ & $\begin{array}{l}5 \text { Degree } \\
\text { Of } \\
\text { Freedom- } \\
5 \text { AX-12 } \\
\text { connected } \\
\text { by daisy } \\
\text { chain }\end{array}$ & $\begin{array}{c}\text { Hokuyo } \\
\text { URG- } \\
\text { 04LX- } \\
\text { UG01 }\end{array}$ & $\begin{array}{c}5 " \\
\text { BaneBots } \\
\text { wheels } \\
\text { connected } \\
\text { with } \\
\text { Pololu } \\
\text { 100:1 } 220 \\
\text { oz-in } 100 \\
\text { RPM } \\
\text { drive } \\
\text { motors. }\end{array}$ \\
\hline Wubble & $\begin{array}{c}\text { Videre } \\
\text { Stereo } \\
\text { camera } \\
\text { with } 2 \mathrm{AX}- \\
12 \text { for } \\
\text { panning } \\
\text { and Tilting }\end{array}$ & $\begin{array}{l}5 \text { Degree } \\
\text { Of } \\
\text { Freedom- } \\
\text { Crustcraw } \\
\text { ler Smart } \\
\text { arm }\end{array}$ & $\begin{array}{c}\text { Hokuyo } \\
\text { URG- } \\
\text { 04LX- } \\
\text { UG01 }\end{array}$ & $\begin{array}{l}\text { Videre } \\
\text { ERRATI } \\
\text { C (Mobile } \\
\text { Base) }\end{array}$ \\
\hline ATOM & $\begin{array}{l}\text { Mirosoft } \\
\text { Kinect and } \\
\text { Custom } \\
\text { Stereoscopi } \\
\text { c Camera } \\
\text { with } 2 \mathrm{AX}- \\
12 \text { for } \\
\text { panning } \\
\text { and Tilting }\end{array}$ & $\begin{array}{c}4 \text { Degree } \\
\text { Of } \\
\text { Freedom- } \\
1 \mathrm{AX}-12 \\
\text { for } \\
\text { shoulder_- } \\
\text { pan, } 2 \\
\text { AX-12 for } \\
\text { shoulder_1 } \\
\text { ift, } 2 \mathrm{AX}- \\
12 \text { for } \\
\text { wrist_flex } \\
1 \text { AX-12 } \\
\text { for } \\
\text { wrist_roll }\end{array}$ & $\begin{array}{c}\text { Microsoft } \\
\text { kinect as } \\
\text { base_kine } \\
\text { ct }\end{array}$ & $\begin{array}{l}4 " \text { wheels } \\
\text { connected } \\
\text { two 30:1 } \\
\text { 85RPM } \\
\text { 37DL } \\
\text { Gear } \\
\text { Motors } \\
\text { with Shaft } \\
\text { Encoder }\end{array}$ \\
\hline
\end{tabular}

\section{Conclusion}

We believe that this paper would be highly beneficial for those robotics engineers and hobby roboticists who wish to create a technologically sound robotic module but staggers in doing so because of the high prices of the cutting edge technology. As we have already gone through those circumstances we realize the pain and eagerness in doing so. We promise to publish our codes of ATOM on the open-source robotics community for people who want to reuse the codes into their own modules. ROS provides a very welldefined framework for implementation. Users can easily get the touch of working on a high end module by implementing their codes on Gazebo. But implementation of ROS on a practical real time platform is always a good practice.

\section{Acknowledgement}

The authors would like to acknowledge Indian Institute of Information Technology, Allahabad for partially funding the initiative. We like to thanks Mrs.Debashree Makhal for her glorious encouragement and appreciations which made us capable of transforing this dream into reality. We appreciate the works done by R.Patrick Goebel on Pi-robot, Dr. Rainer Hessmer on ARDROS, Alan Downing for PR2-Lite and Willow Garage software engineer Michael Ferguson on Maxewll. We thank them for excellent documentation which made our work easy.

\section{REFERENCES}

[1] "The Uncanny Valley" Masahiro Mori, 1970, Energy, 7(4), pp. 33-35 Translated by Karl F. MacDorman and Takashi Minato.

[2] Quigley, Morgan., Conley, Ken., Gerkey, Brian P.., Faust, Josh., Foote, Tully., Leibs, Jeremy., Wheeler, Rob., and Ng, Andrew Y.;" ROS: an open-source Robot Operating System" ICRA Workshop on Open Source Software, 2009

[3] http://www.ros.org/wiki/Robots/PR2 \{Till date $12 / 12 / 2012\}$

[4] http://www.care-o-bot-research.org/care-o-bot-3 date $12 / 12 / 2012$ \}

[5] R.D. Schraft and C. Schaeffer and T. May," Care-O-bot: The Concept of a System for Assisting Elderly or Disabled Persons in Home Environments" Proceedings of the 24th Annual Conference of the IEEE,1998

[6] http://thearmrobot.com/ \{Till date 12/12/2012\}

[7] Cognition-Enabled Autonomous Robot Control for the Realization of Home Chore Task Intelligence (Michael Beetz, Dominik Jain, Lorenz Mösenlechner, Moritz Tenorth, Lars Kunze, Nico Blodow, Dejan Pangercic), In Proceedings of the IEEE, volume 100, 2012.

[8] http://stair.stanford.edu/ \{Till date 12/12/2012\}

[9] Siddhartha S.Srinivasa,Dave Ferguson,Casey J.Helfrich, Dmitry Berenson, Alvaro Collet, Rosen Diankov, Garrat Gallagher, Geoffrey Hollinger, James Kuffner, Michael VandeWeghe "Herb: A home exploring robotic butler", Autonomous Robots, 2009

[10] http://personalrobotics.ri.cmu.edu/projects/herb.php \{Till date 12/12/2012\}

[11] http://www.showusyoursensors.com/ $12 / 12 / 2012\}$

[12] http://www.ros.org/wiki/maxwell \{Till date 12/12/2012 \}

[13] http://www.pirobot.org/ \{Till date 12/12/2012\}

[14] http://ros.org/wiki/Robots/TurtleBot \{Till date $12 / 12 / 2012\}$

[15] http://streebgreebling.blogspot.in/2010/04/poor-manrlaser-scanner.html \{Till date 12/12/2012\}

[16] http://code.google.com/p/ua-ros-pkg/wiki/WubbleRobot

\{Till date $12 / 12 / 2012\}$ 